\title{
EDITORIAL
}

\section{¡Europa!, a pesar de todo. Una estrategia realista}

\section{Consejo de Redacción}

Resumen: Europa resiste, a pesar de todo. Aunque en un pasado no demasiado lejano un par de referéndum en Francia y en los Países Bajos sirvió para bloquear el proceso de ratificación del proyecto de Constitución europea, éste fue posteriormente reconducido al posterior Tratado de Lisboa, en que se recogieron los aspectos más sustanciales del fracasado proyecto constitucional.

Los apocalípticos creen que el llamado "brexit" es el preludio de la inmediata disolución de la UE, incapaz de hacer frente a la crisis económica y sus consecuencias sociales y ante los desafíos de la nueva realidad mundial y la hegemonía emergente de grandes potencias.

El acontecimiento del "brexit" ha despertado las conciencias de los europeos. Lo que queda es la imagen de una UE cuajada de perfiles negativos: déficit democrático estructural, altiva burocracia, instituciones bloqueadas, procesos complejos de codecisión y una oleada de populismos de distinto signo que sólo persiguen la destrucción del sistema de la Unión Europea.

Para los críticos y los más apocalípticos, desde un punto de vista geoestratégico Europa sería un perdedor neto de la "hiperglobalización", pues el eje franco-alemán hace tiempo que dejó de funcionar, lo que unido a su falta de democracia y de transparencia y su incapacidad para enfrentar la principal catástrofe humanitaria de los últimos tiempos, la oleada de refugiados sirios, haría que nos preguntásemos si podemos seguir afirmando que esto es lo normal y previsible, pues la construcción europea siempre ha salido adelante de sucesivas y periódicas crisis con imaginación.

Después de describir algunos elementos de la actual crisis de la UE, el editorial plantea la pregunta: ¿̇han cambiado los valores fundacionales de la UE? Nuestro editorial se inscribe en una reflexión sobre Europa de esta revista iniciada hace años (los títulos de los editoriales figuran en un cuadro final). Hace cinco años nos pronunciábamos sobre la construcción europea a favor de una consolidación, desarrolloy profundización del sistema constitucional europeo de Economía social de mercado. Propugnábamos entonces resocializar el proyecto europeo con nuevas energías, con nueva claridad, con nueva pasión europeísta. Para salir 
del actual bloqueo, la UE debe liberarse de ese único modelo de capitalismo neoliberal que está precisamente en el origen de la crisis. A continuación de la introducción, abordamos las instituciones europeas y sus resultados, los producidos por sus políticas agrarias y de cohesión regional, muchas veces ignoradas o silenciadas. No silenciamos, por supuesto, los aspectos más problemáticos o negativos, especialmente la insuficiente respuesta europea ante la grave crisis humanitaria siria que nos pone en evidencia ante el mundo y ante nosotros mismos. Si la capacidad de los gobiernos de las potencias para responder a grandes crisis es tan limitada como se demuestra en estos tiempos, la UE no es una excepción. En el apartado cuarto, sin embargo, presentamos los desafíos con los que se encuentra la UE, con alguna referencia a las políticas españolas desarrolladas a partir del acervo comunitario.

El quinto apartado y conclusión nos permite afirmar que la UE es una realidad institucional plenamente consolidada desde hace tiempo, un sistema de economía social de mercado altamente competitiva y solidaria y una aceptable capacidad de insertarse desde un sistema de gobernanza democrática en un mundo hiperglobalizado. Europa puede conjugar una mundialización moderada, la democracia como sistema y la permanencia de los Estados. Nuestra convicción es ésta: la UE está ante una única estrategia realista posible y debe apostar a fondo por ella. Por ello, a pesar de todo ¡Europa!

Palabras clave: globalización moderada, gobernanza multinivel, hiperglobalización, Unión Europea.

Fecha de aprobación: 18 de abril de 2017.

\section{Europe against all odds! A realistic strategy}

Abstracts: Europe resists against all odds. Although, in the not-so-distant past, a couple of referenda in France and the Netherlands succeeded in blocking the process of ratifying the draft European Constitution, this was then redirected to the subsequent Lisbon Treaty, in which the most significant aspects of the failed constitutional project were presented.

The apocalyptic believe that the so called "brexit" is the prelude to the immediate dilution of the EU, unable to face the economic crisis and its social consequences, the challenges of the new global reality and the rising hegemony of big powers.

\section{L'Europe malgré tout. Une stratégie réaliste}

Résumé: L'Europe résiste malgré tout. Malgré le fait que dans un passé non lointain deux référendums, qui avaient eu lieu en France et aux Pays-Bas, avaient servi à bloquer le processus de ratification du projet de la Constitution Européenne, celui-ci a été postérieurement repris dans le Traité de Lisbonne, dans lequel sont présents les aspects les plus essentiels du projet constitutionnel ayant échové.

Les «apocalyptiques» croient que le «brexit» estle prélude d'une très prochaine disparition de l'UE, incapable de faire face à la crise économique et à ses conséquences sociales, 
The "brexit" event has awoken the consciousness of European citizens. What remains is a picture of the EU imbued with negative outlines: structural democratic deficit, haughty bureaucracy, blocked institutions, complex codecision processes and a rising tide of populisms of different political hues which solely seek the destruction of the European Union system.

According to the strongest critics and the most apocalyptic, geostrategically Europe would be the major net loser of the "hyperglobalisation", as the French-German axis ceased to function long ago. Along with this, the lack of democracy and transparency, and their inability to successfully face the main humanitarian catastrophe of recent times, the wave of Syrian refugees, would lead us to wonder whether we can still maintain that this is normal and foreseeable, as the European construction has always found an imaginative way out of successive and recurrent crises.

After describing some of the elements of the current EU crisis, the editorial raises the following question: have the EU founding values changed? Our editorial comes within a reflection on Europe that was started years ago in this journal (the titles for the different editorials are shown in a table at the end). Five years ago, we spoke in favour of the development and deepening of the constitutional European system of a social market economy. We advocated the resocialisation of the European project with new energies, new clarity and new passion for Europe. In order to find out a way out of the current impasse, the EU should get away from that single model of neoliberal capitalism which is precisely at the origin of the crisis. Following the introduction, we examined the European institutions and their results, produced by their most of the times ignored or silenced ainsi qu'aux défis de la nouvelle réalité mondiale et à l'hégémonie émergente de certaines grandes puissances.

L'événement marquant du «brexit» a provoqué une prise de conscience des Européens. $\mathrm{Ce}$ qui en reste est l'image d'une Union Européenne remplie $d$ 'aspects négatifs: déficit démocratique structurel, bureaucratie hautaine, institutions bloquées, processus complexes de prise de décisions et une vague de populismes de différents bords qui ne cherchent que la destruction du système de I'Union Européenne.

Pour les critiques et les plus apocalyptiques, L'Europe serait, d'un point de vue géostratégique, un net perdant de «l'hyper-mondialisation», puisque l'axe franco-allemand ne marche plus depuis longtemps. Cela, ajouté à son manque de démocratie et de transparence et à son incapacité à faire face à la catastrophe humanitaire des réfugiés syriens, pourrait nous faire nous demander si la construction européenne sera capable de s'en sortir avec l'imagination dont elle a fait preuve après les successives et périodiques crises qu'elle a vécues.

Après la description de quelques éléments de la crise actuelle de l'UE, l'éditorial pose la question suivante : les valeurs fondatrices de l'Union Européenne ont-elles changé ? Notre éditorial s'inscrit dans la réflexion de cette revue sur l'Europe, commencée il y a quelques années (les titres des éditoriaux se trouvent dans le tableau récapitulatif final). Il y a cinq ans nous sommes prononcés sur la construction européenne, en faveur de la consolidation, le développement et l'approfondissement du système constitutionnel européen d'Économie sociale de marché. Nous avons alors voulu promouvoir la resocialisation du projet européen avec de 
agricultural and social cohesion policies. We do not omit, of course, the most problematic or negative issues, particularly the insufficient European response to the serious humanitarian crisis, which has exposed us to the whole world and to ourselves. If the capacity of governments to response to great crises is as limited as shown in these times, the EU is not an exception. Nevertheless, we will present in part four the challenges that the EU is facing, with some references to the Spanish policies developed on the basis of the acquis communautaire.

Part five and conclusion enables us to state that the EU is a long, well-established institutional reality, a highly competitive social market economy geared to solidarity, and with an acceptable capacity to insert itself from a democratic governance system into a hyperglobalised world. Europe can combine a moderate globalization, democracy as system and the permanence of the Member States. This is our firm belief: the EU is facing the only possible strategy and should opt fully for it. For this reason and against all odds, Europe!

Key words: moderate globalisation, multilevel governance, hyperglobalisation, European Union. nouvelles énergies, avec une nouvelle clarté et une nouvelle passion tournée vers l'Europe. Pour sortir de l'impasse actuelle, L'UE doit se libérer de cet unique modèle de capitalisme néolibéral qui est précisément à l'origine de la crise actuelle. Après l'introduction, nous abordons les institutions européennes et leurs résultats, ainsi que ceux produits par les politiques agraires et de cohésion régionale, souvent ignorées ou réduites au silence. Bien sûr, nous n'oublions pas les aspects les plus problématiques ou négatifs, plus particulièrement la réponse insuffisante de l'Europe face à la grave crise humanitaire syrienne, qui nous remet en cause face au monde et face à nous-mêmes. Si la capacité de réponse des gouvernements des grandes puissances face aux grandes crises est aussi limitée, comme nous pouvons le remarquer actuellement, l'UE n'en est pas une exception. Cependant, dans la quatrième partie, nous présentons les défis auxquels l'Europe doit faire face, avec quelques références aux politiques espagnoles développées à partir de l'acquis communautaire.

Dans la cinquième partie et dans la conclusion, nous affirmons que l'UE est une réalité institutionnelle pleinement consolidée depuis longtemps, un système d'économie sociale de marché hautement compétitif et solidaire et qui est capable de s'insérer dans un système de gouvernance démocratique dans un monde hyper-mondialisé. L'Europe peut conjuguer une mondialisation modérée, une démocratie comme système et le maintien des États. Voici notre conviction : L'UE est face à une stratégie réaliste possible et doit miser pleinement sur elle. C'est pour cela que nous pouvons dire, malgré tout, L'Europe!

Mots clé: mondialisation modérée, gouvernance multi-niveaux, hyper-mondialisation, Union européenne. 


\section{Introducción: la crisis}

Parece que fue el viernes 24 de junio de 2016 el día de la catástrofe: con un 51,9 $\%$ de los votos, el sector más conservador de la sociedad inglesa (en el que predominaban las personas mayores de edad, los habitantes de regiones y ciudades desindustrializadas y los habitantes de zonas rurales) consiguió una victoria que nadie esperaba: la salida del Reino Unido de la Unión Europea (a partir de ahora UE). El diccionario Oxford ha elegido como palabra del año post-truth (posverdad). Éste es un concepto híbrido y ambiguo que quiere expresar que en la formación de la opinión pública los hechos objetivos influyen menos que los llamamientos emocionales. Un ejemplo de posverdad ha sido el, que nadie previó aunque algunos lo temieran, pero que se impuso contra las evidencias de lo razonable. Podríamos considerarlo un ejemplo de la política emotiva a la que recientemente nos referimos en estas páginas'. Los llamamientos a referéndum generan efectos paradóiicos e imprevistos: en esta ocasión fue convocado, por el premier David Cameron al parecer con el único objetivo de tratar de resolver tensiones internas en su propio partido conservador, contando con el apoyo del fantasmagórico partido "por la independencia de Reino Unido" (UKIP), aunque podamos recordar que, en el pasado, un referéndum sirviese para bloquear el proceso de ratificación del proyecto de Constitución Europea (en Francia y en los Países Bajos), que se recondujo al posterior Tratado de Lisboa, en que se recogieron los aspectos más sustanciales del anterior y fracasado proyecto de Constitución.

Para algunas voces apocalípticas, el impacto imprevisto del "brexit" vendría a ser como el preludio de la próxima o inmediata disolución de una UE cada día más débil e inconsistente, que se ha demostrado incapaz de hacer frente a la crisis económica y sus consecuencias sociales, y que no ha conseguido asumir un papel relevante ante los desafíos de una nueva realidad mundial condicionada por la emergencia de grandes potencias con políticas fuertes, como China, EE.UU. o Rusia.

Ante la sorpresa del cataclismo imprevisto, casi nadie se ha parado a recordar que precisamente el Reino Unido durante las últimas décadas ha sido el gran freno histórico al proceso de consolidación europea; que se ha comportado reiteradamente como el factor discordante de Bruselas, limitando, bloqueando y coartando cuantas iniciativas de consolidación y reforzamiento de la UE se han ido proponiendo, operando en la práctica como un socio desleal y desconfiado. Entendemos que una organización tan compleja como la UE no puede subsistir

' CONSEJO DE REDACCIÓN (2015) "La democracia al servicio de todos, integrante del bien común": Revista de Fomento Social 70. 7-28 (18).

Revista de Fomento Social 72/2 (20|7) 
a largo plazo contando con socios que sirvan de freno u obstáculo a su propio desarrollo, pues no comparten sus valores.

Por otra parte, sorprendentemente, el primer acuerdo sobre el "brexit" se ha tomado por unanimidad; jnada de divergencias que puedan poner en riesgo a la UE... por ahora! Es cierto que el proceso de salida del Reino Unido será complejo y dará lugar, sin duda, a problemas difíciles de resolver.

Sin embargo, lo cierto es que el acontecimiento ha servido en la práctica para levantar todos los vientos apocalípticos que desde hace tiempo soplaban en contra de la UE. Lo que queda al final es una imagen cuajada de perfiles en negativo: una organización inmersa en un déficit democrático estructural, dirigida por una altiva burocracia de Bruselas, con unas instituciones bloqueadas cotidianamente ante procesos de codecisión tortuosos y complejos, incapaz de asegurar unas políticas de cohesión que rompan con la creciente tensión norte/sur, que cometió en el pasado la imprudencia de sumergirse en una unión monetaria sin asegurar previamente unas pautas mínimas de homogeneización fiscal o macroeconómica, y que incluso en la práctica parece incapaz de evitar la existencia de paraísos fiscales interiores (en primer lugar Irlanda, peor también Luxemburgo y en cierta manera los Países Bajos, ambos Estados fundadores) permitiendo un reiterado "dumping" fiscal claramente contrario a la propia lógica inspiradora de la UE. Una organización, en fin, crecientemente asolada por una oleada de populismos de distinto signo que sólo persiguen la destrucción de Europa.

Parece que una vez abierta la veda, todos se apresuran a disparar al blanco fácil:

Europa, tal como fue soñada, se acabó. Se ha convertido en el templo de unos burócratas que, a consecuencia del dominio y del poderío alemán, dejan morir el sueño europeo en medio de unos programas de austeridad salvajes... (NAVALón, El País, 2017);

...tras el fiasco-casi un engaño-del supuesto Tratado Constitucional, y la posterior puesta en marcha del de Lisboa, y aún reponiéndose de la última ampliación, la UE camina a paso lento, arrastrando los pies, sin liderazgo político y sin acabar de tener un papel claro en el nuevo orden mundial (LAMO DE ESPINOSA, Instituto Elcano, 2016);

La Unión Europea ha perdido el grueso de sus ilusiones y mitos fundadores. La crisis financiera de 2007/2008 ha demostrado que no es un club democrático de iguales, sino una construcción oligárquica y antidemocrática (POCH DE FELIU, La Vanguardia, 2016).

La serie argumentativa podría reproducirse hasta el infinito: desde una perspectiva geoestratégica Europa sería un perdedor neto de la actual "hiperglobalización" montada sobre los nuevos imperios. Su principal plataforma de impulso (el famoso eje franco-alemán) hace tiempo que habría dejado de funcionar. Parece inmersa 
en un marasmo de "balcanización" que se alimenta de su falta de democracia y de transparencia. Y además, para colmo, ha caído en el peligroso juego de buscar nuevos enemigos (Rusia) después de integrar a los países del este ofreciéndoles formar parte de un bloque militar anti-ruso como paso previo a su ingreso en la UE, lo que abre ahora las puertas al sordo y amenazante conflicto de Ucrania. Estamos ante un cúmulo de desdichas que alcanzan su punto culminante ante la incapacidad demostrada para enfrentar la principal catástrofe humanitaria de los últimos tiempos: la oleada de refugiados que huyen del conflicto sirio².

Aunque constituye un argumento reiterado la afirmación de que la construcción europea se ha desarrollado históricamente con sucesivas y periódicas crisis que siempre han ido suscitando la búsqueda de salidas imaginativas, ante esta vorágine apocalíptica conviene comenzar recordando lo más elemental. ${ }^{3}$

Una organización, de cualquier tipo, se define por una visión, una misión y unos valores. Si las primeras, misión y visión, van cambiando en un proceso permanente de adaptación al entorno o de cambios en el mismo, los valores que la definen son más estables y tienen vocación de permanencia. Son los valores los que identifican a la organización; si aquéllos cambian, es ésta la que está cambiando. Dirigir una organización hacia el futuro implica mantener la coherencia de la misión, de la visión y de los valores. A partir de este principio podríamos preguntarnos: ¿̇han cambiado los valores fundacionales de la UE?

Hace cinco años, nos pronunciábamos en otro editorial ${ }^{4}$ sobre la construcción europea y lo hacíamos a favor de una consolidación, desarrollo y profundización del sistema constitucional europeo de Economía social de mercado y de su establecimiento formal con valor normativo para integrar las aspiraciones europeas más auténticas y elevadas: la solidaridad, la responsabilidad y la productividad. Europa sólo podrá salir de su profunda y persistente crisis si no pierde su alma, escribíamos entonces, propugnando un ejercicio de ciudadanía para resocializar el proyecto europeo con nuevas energías, con nueva claridad, con nueva pasión

${ }^{2}$ Cf. CONSEJO DE REDACCIÓN (2016) "Puertas abiertas / puertas cerradas: los refugiados ponen en evidencia a Europa": Revista de Fomento Social 71, 431-454.

${ }^{3}$ Una descripción y análisis de la crisis de la UE muy clara y asequible para todo lector, que compartimos, es la que ofrece nuestro compañero de la Universidad Loyola Andalucía profesor Pedro CALDENTEY DEL Pozo (2016) "La Unión Europea en crisis": Seguridad y sociedad 6, 47-52 (n. 13, junio).

${ }^{4}$ CONSEjO de RedACCIÓn (2012) "Para una Europa solidaria, responsable y productiva, un sistema de Economía social de mercado": Revista de Fomento Social 67, 5-29. 
europeísta. La actual situación europea es tal que sólo con ese cambio profundo, decíamos, podremos salir del actual bloqueo. Frente a los que hubiesen preferido una convergencia hacia un único modelo de capitalismo neoliberal que está precisamente en el origen de la crisis defendimos nuestro sistema europeo constitucional porque creíamos que esa era la forma de ser más europeo.

Entonces nos planteábamos la tensión existente, que no ha hecho más que agrandarse, entre democracia nacional y mercados globales, ante lo que esbozábamos tres posibilidades: la de limitar la democracia con el propósito de minimizar los costes de transacción internacionales, sin tener en cuenta los trastornos económicos y sociales que la economía global produce en ocasiones, la de limitar la globalización con la esperanza de reforzar la legitimidad democrática en cada país y la de globalizar la democracia a costa de la soberanía nacional. Lo que nos parecía evidente era que todo a la vez no era posible: hiperglobalización, democracia y autodeterminación nacional. Si queremos hiperglobalización y democracia, tenemos que renunciar al Estado-nación, si hemos de mantener el Estado-nación con la hiperglobalización, tendremos que olvidarnos de la democracia y, si queremos disponer de un Estado-nación con democracia, una globalización profunda no es posible. Ésta es precisamente, según Rodrik, la paradoja de la globalización. Por ahora una gobernanza global democrática es sencillamente imposible. Un mundo con una globalización moderada sería un lugar mucho mejor para vivir, que uno atrapado en la quimérica búsqueda de la hiperglobalización. Ese autor admitía muy significativamente la única excepción de la UE, que confirmaría la regla. Europa podía conjugar una mundialización o globalización al menos moderada, la democracia como sistema y la permanencia de los Estados nacionales. El debate francés, cuando escribimos estas líneas, entre protección estatal y apertura mundial, ilustra bien la agudeza de la cuestión, al mismo tiempo que la convicción, que mantenemos, de que la UE es la única excepción a la famosa aporía de Rodrik, como él mismo apuntó, y la única estrategia realista posible. ${ }^{5}$

\section{Las instituciones}

El sistema institucional de la actual UE se configura a partir del mandato surgido de las elecciones al Parlamento Europeo de 2014, cuando por primera vez se estableció la exigencia de que el presidente de la Comisión fuera el candidato del partido vencedor en los comicios: una contundente muestra de democracia parla-

${ }^{5}$ Cf. D. RodRIK (2011) La paradoja de la globalización, Barcelona, Antoni Bosch, 366. 
mentaria que vino acompañada de la habitual comparecencia ante el Parlamento europeo de todos y cada uno de los candidatos a Comisario, quienes además de recibir su aprobación individual requirieron también de la votación final del Parlamento sobre el conjunto de la Comisión. En rigor, podemos afirmar que no existe un solo Estado miembro de la UE en que el ejecutivo se someta a tal grado de control democrático.

Naturalmente cualquiera podría argüir que las elecciones al Parlamento europeo son, en la práctica, elecciones "de segundo orden", en las que el electorado realmente no tiene una capacidad de decisión representativa similar a la que se produce en las elecciones nacionales: a lo que convendría replicar que, al menos en España, las elecciones europeas resultan ser las únicas auténticamente proporcionales puesto que no se utilizan circunscripciones electorales provinciales. Por otra parte, elecciones de segundo orden en la escala territorial multinivel, local o regional, se producen en todos los países europeos.

Pero con independencia de los factores de tipo técnico-representativo, se viene insistiendo, más en general, en que Europa en realidad es una pura suma de Estados lingüística y culturalmente diversos, que carece de un único "démos" capaz de legitimar un sistema institucional unitario auténticamente representativo. Es curioso que unos meses después del "brexit", a comienzos de enero de 2017, haya tenido lugar el lanzamiento de una campaña por parte de la UE para conmemorar el trigésimo aniversario de "Erasmus", el famoso programa de intercambio de estudiantes entre universidades europeas. Se calcula en un total de cinco millones de estudiantes la cifra de beneficiarios de este programa que, debido a su alta valoración, se considera que ha contribuido decisivamente a formar a la nueva generación europea que parece adoptar actitudes bien alejadas de los viejos nacionalismos y que se configura como el soporte cosmopolita de una futura población de ciudadanos europeos.

En cualquier caso ha sido esa ciudadanía europea, aunque en formación, la que ha elegido un Parlamento que viene manifestando un dinamismo creciente como foro de debate y como instrumento de control de la Comisión. Pese a las dudas iniciales, los procedimientos de codecisión de las instituciones de la UE están demostrando un alto grado de operatividad: el Consejo europeo, que actúa a modo de "Jefe de Estado", cuenta con un presidente permanente, en la actualidad el polaco Donald Tusk, y determina junto con la Comisión la agenda de iniciativas legislativas que tanto el Parlamento como el Consejo, a modo de segunda cámara formada por los ministros del ramo en cuestión en cada Estado, debaten y aprueban. Las materias ajenas a este procedimiento se han reducido sustancialmente. En consecuencia, 
la labor legislativa y de control del Parlamento europeo se viene consolidando de un modo similar a cualquier otra democracia parlamentaria.

$Y$, como sucede en las modernas democracias, hay también una serie de instituciones independientes que, a modo de agencias, desempeñan funciones especializadas. No cabe calificar a tales agencias como antidemocráticas puesto que sus dirigentes son nombrados por gobiernos e instituciones democráticas, contando con el requisito de la cualificación técnica o el conocimiento experto. Y recordemos que a veces han actuado con notable éxito: baste mencionar la fulminante respuesta del presidente del Banco central europeo (BCE) Mario Draghi durante la grave crisis de deuda desencadenada, cuando en julio de 2012 declaró su voluntad de hacer todo lo necesario para salvaguardar el euro, añadiendo su célebre frase ... and believe me, it will be enough, lo que produjo un impacto inmediato sobre las bolsas que terminó con la angustiosa sensación de incertidumbre que asolaba a algunos países europeos, singularmente a España.

Ciertamente, se ha criticado que los países miembros de la UE, en sus diversas y complejas estrategias de respuesta a la crisis, hayan optado a veces por crear instrumentos o mecanismos ajenos al propio organigrama de la UE, como el "Eurogrupo", el Mecanismo de estabilidad financiera (MEDE) o la denominada "troika" (integrada por delegaciones de los Estados miembros, el Fondo Monetario Internacional y el propio BCE). Se olvida a veces la elemental constatación de que, aunque estas instituciones $O$ instrumentos no sean parte formalmente del organigrama de la UE, son sin embargo instituciones que han desarrollado estrategias de rescate de los países europeos, donde con frecuencia los propios parlamentos de los Estados que han aportado financiación a esos fondos, desarrollan funciones de control. En otros supuestos se ha tratado en cambio de mecanismos que refuerzan el papel de las instituciones de la UE, como los famosos "paquetes" (el "Six Pack" de 2011 y el "Two Pack" de 2013) donde se establece nada menos que el control de la Comisión sobre los presupuestos de los Estados incluso antes de su aprobación por los propios parlamentos estatales.

Pero más allá del organigrama formal de las instituciones de la UE se encuentran sus propios recursos humanos, donde se alude reiteradamente al tópico de la arrogante burocracia de Bruselas como fuente de todos los males: una organización que se percibe como un bloque elitista y tecnocrático, alejado del tejido social, que trabaja bajo un manto de opacidad con criterios y argumentos sofisticados perfectamente ajenos a los problemas cotidianos de la gente de la calle. Es una lástima que se ignore demasiado frecuentemente el alto grado de transparencia que preside sus actuaciones, los hábitos participativos que orientan su labor, puesto que no hay 
programa o propuesta que no haya contado previamente con la participación de las redes de actores, o incluso el sofisticado mecanismo de evaluación previa a que se someten todas sus propuestas antes de recibir la aprobación formal de la Comisión, que en el pasado año 2016 alcanzó hasta sesenta y tres evaluaciones de impacto previas: una innovación real, que acaso mereciera ser aplicada a los gobiernos de los Estados.

La burocracia de la UE podría ser calificada, sin exagerar, como la mejor burocracia del mundo, por más que el éxito de su actuación dependa al final, naturalmente, de la propia actuación de las distintas burocracias estatales, precisamente el lugar donde suelen producirse los desajustes, las deficiencias, las corruptelas o los incumplimientos.

\section{Los resultados}

Pero con independencia de las posibles valoraciones positivas del sistema institucional de la UE, al final de lo que se trata es de comprobar sus resultados y, en particular, la difícil travesía sobre la gran crisis desencadenada en 2008.

En primer lugar, convendría recordar los más que aceptables, y muchas veces silenciados, resultados europeos en varias de sus políticas: las agrarias por un lado y las de cohesión regional por otro. Más que problemáticos, sin embargo, son el déficit en coherencia de las políticas fiscales y el déficit en ciertos aspectos de las políticas monetarias.

Se ha reiterado hasta la saciedad el argumento de que la estrategia económica europea ha adolecido en general de un deficiente enfoque, inspirado en una filosofía de tipo neoliberal que se ha demostrado inadecuada en momentos de ciclo depresivo, a diferencia de la estrategia seguida por la Administración Obama en EE. UU. Es lo que en expresión gráfica se ha dado en denominar "austericidio". No pretendemos tratar de confirmar aquí si se trata o no de una estrategia errónea, para lo que nos remitimos a anteriores editoriales citados, pero lo que no cabe es dejar de reconocer que tal estrategia constituye una expresión congruente de los perfiles políticos e ideológicos manifestados por la mayoría de la población europea; es decir, responde a elementales claves democráticas.

Recordemos que la composición actual del Parlamento europeo refleja una mayoría del grupo conservador (popular) y una clara hegemonía de las fuerzas de centroderecha, integrada por diferentes partidos conservadores, populares y liberales. 
Excluyendo minorías y sectores radicales, el sector de centro-derecha ascendería a casi el $48 \%$ de la representación parlamentaria, frente a un 39\% de la izquierda, incluyendo los Verdes. Un mapa que reproduce, como es lógico, la situación política interna de los distintos Estados, y que se expresa también en el Consejo de Europa. Salvo la dinámica inercial de Italia o Francia, sólo han aparecido "nuevos" gobiernos de izquierdas en Grecia y en Portugal, aunque en el segundo caso con un grupo parlamentario mayoritario de derechas (102 escaños frente a los 86 escaños del partido socialista que ejerce la presidencia del gobierno). En algunos países se mantienen grandes coaliciones, mientras que la mayoría de las recientes elecciones han dado lugar en los últimos años a gobiernos de centro derecha, como en Hungría, Dinamarca, Polonia, Estonia o Finlandia.

Extrañarse de que, ante semejante panorama político, la UE desarrolle políticas económicas conservadoras se debe a cierta ceguera política. Otro asunto más grave es que, como consecuencia de tales políticas, se haya desarrollado una sorda tensión norte/sur que contradice al propio espíritu fundacional de la UE y que, por más que puedan detectarse síntomas de recuperación en los países mediterráneos, con España a la cabeza, esconde a veces prejuicios culturales como el que recientemente expresaba con sus extemporáneas declaraciones el holandés presidente del "Eurogrupo" Jeroen Dijsselbloem. Un dualismo norte/sur sobre el que todavía cabalgan viejos fantasmas como el que trata de enfrentar el calvinismo del norte frente al catolicismo del sur y que ya se pusieron de manifiesto a comienzos del XX, años en que escribió la famosa ética protestante y el espíritu del capitalismo de Max Weber, publicada en 1905, y ya entonces cuestionada por algunos historiadores.

Naturalmente, más allá de la propia crisis económica, están sus graves consecuencias sociales, que han puesto a prueba la capacidad de resistencia y amortiguación de los distintos Estados sociales y sus políticas asistenciales, afectadas por la inexorable escasez de fondos públicos; unas políticas que, recordemos, no están en manos de la UE, sino en las de los diferentes Estados. Por supuesto, como corresponde a un contexto democrático, las víctimas de la crisis no han dejado de encontrar cauces para manifestar su protesta y tales protestas y movilizaciones han acabado convirtiéndose al final en el mejor caldo de cultivo de movimientos radicales o populistas de distinto signo que parecen expandirse de forma desigual: desde el nihilismo antisistema del italiano "Movimiento Cinque Stelle" hasta el permanente crecimiento del derechista "Front National" dirigido por Marine Le Pen en Francia. Son los casos más próximos o conocidos, aunque perfiles populistas presentan otras fuerzas políticas de extrema derecha, como los "Verdaderos Finlandeses", que forman parte de un gobierno en coalición, el "Partido del Pueblo Danés", el 
"Fidesz" húngaro de Viktor Orban, en el gobierno, el también gobernante "Ley y Justicia" (PiS) polaco, el austríaco "Partido de la Libertad" (FPÔ), la "Alternativa para Alemania" (AfD), el "Partido por la Libertad" (PVV) de Geert Wilders en los Países Bajos o "Amanecer Dorado" en Grecia.

¿Dispone la Unión Europea de algún instrumento de respuesta ante semejante amenaza? Evidentemente sí, pues el artículo séptimo del Tratado de Lisboa prevé la suspensión de los derechos de pertenencia para aquellos Estados que violen los valores contemplados en el artículo segundo ${ }^{6}$. Éste es un mecanismo que hasta ahora se ha utilizado en los casos de Austria, Polonia y Hungría como amenaza o bien mediante una aplicación temporal, lo que significa pues que la propia UE se configura como una barrera defensiva frente a los riesgos de degradación democrática o de ruptura del civilizado marco del Estado de Derecho.

Más grave sería acaso la insuficiente respuesta a la terrible crisis humana de los refugiados sirios ${ }^{7}$, en especial si consideramos que la ruta terrestre natural por la que éstos deben desplazarse discurre por la zona balcánica: una región con una persistente memoria histórica de la conflictiva proximidad al imperio otomano así como de las graves consecuencias que siguieron a la traumática disolución de la antigua Yugoslavia. Tampoco puede ignorarse que la opinión pública europea puede acabar siendo lamentablemente afectada por las graves consecuencias de la oleada terrorista desencadenada por el "yihadismo" radical y el terrible impacto de los grandes atentados producidos en algunas de las principales capitales europeas. Por más que el papa Francisco haya reiterado sus llamamientos a la paz, el resultado final parece claramente insatisfactorio, pues hasta ahora la única y precaria respuesta parece haber sido el apoyo a la posición de Turquía como espacio de amortiguación del movimiento migratorio. Es evidente que los europeos deberíamos hacer mucho más y que los instrumentos de coordinación en este ámbito han funcionado muy deficientemente.

Un balance razonable reflejaría pues la conclusión de que la Unión Europea no es una institución con capacidad suficiente para hacer frente a crisis de gran calado

\footnotetext{
- La Unión se fundamenta en los valores de respeto de la dignidad humana, libertad, democracia, igualdad, Estado de Derecho y respeto de los derechos humanos, incluidos los derechos de las personas pertenecientes a minorías. Estos valores son comunes a los Estados miembros en una sociedad caracterizada por el pluralismo, la no discriminación, la tolerancia, la justicia, la solidaridad y la igualdad entre mujeres y hombres.

${ }^{7}$ Cf. Nuestro anterior editorial: CONSEJO DE REDACCIÓN (2016) "Puertas abiertas / puertas cerradas: los refugiados ponen en evidencia a Europa": Revista de Fomento Social 71, 431-454.
} 
o a emergencias desencadenadas de forma súbita. Aunque se trata de una incapacidad que, en el fondo, también afecta a los propios Estados. Por desgracia la experiencia histórica viene demostrando que el recurso a los estados de excepción no es una solución satisfactoria. Aunque resulte razonable suponer que, en momentos de grave crisis e incertidumbre, los ciudadanos confiemos en la capacidad de respuesta extraordinaria de nuestras instituciones públicas, la realidad parece demostrarnos todo lo contrario, pues todos los sistemas democráticos civilizados parecen operar razonablemente en contextos de relativa normalidad social, pero se demuestran insuficientes cuando se trata de atender a grandes emergencias sobrevenidas que desbordan la capacidad normal de las instituciones.

\section{Los desafíos}

En este contexto parece pues que no deberíamos pedirle a las instituciones de Bruselas una milagrosa capacidad de respuesta contundente, eficaz e inmediata, a las grandes crisis de dimensión mundial desencadenadas a comienzos del siglo XXI. Ni Europa ni Estados Unidos, ni Rusia ni China: la capacidad de los gobiernos para enfrentar grandes crisis sigue siendo limitada y las exigencias de cooperación internacional siguen constituyendo un reiterado desafío en un universo multipolar de creciente complejidad.

En todo caso parece que sólo podremos esperar de la UE una razonable capacidad de mantenimiento y desarrollo del modelo de democracia vigente hasta ahora en nuestro contexto occidental, lo que no es poco. Es aquí donde siguen destacando los valores fundacionales de la Unión, reactualizados en sus distintos documentos constitutivos y reguladores, en los que se muestra la capacidad práctica de innovación en el derecho y en las instituciones europeas a lo largo del tiempo.

Recordemos en primer lugar que la inmensa mayoría de las innovaciones legislativas "estatales" de las últimas décadas han sido en realidad desarrollo del propio derecho europeo, pues en particular hay determinadas áreas o sectores como agricultura, medio ambiente, transporte, calidad alimentaria, regulación de la calidad industrial, etc., donde se constata una clara posición de vanguardia mundial en términos de calidad regulativa. En rigor, las únicas tareas pendientes en este ámbito serían las que se relacionan con la necesidad de asegurar un mejor grado de coordinación entre las diferentes esferas institucionales afectadas: es decir, la llamada gobernanza multinivel, que constituye la principal línea de avance pendiente en este campo; una tarea que se hizo especialmente difícil tras 
la ampliación de la UE a veintiocho miembros. Pero el ámbito de la gobernanza constituye un sector donde no podemos esperar una visualización inmediata de grandes decisiones con un impacto directo sobre la opinión pública, sino más bien una labor sorda y de largo plazo, que implica y compromete activamente al conjunto del sistema social europeo. En la actualidad, la mayor urgencia coordinadora estaría afectando seguramente al ámbito de la seguridad.

Pero como sucede en todo Estado de derecho, la mera actividad normativa o legislativa no es suficiente cuando su proceso de aplicación genera inevitables conflictos; conflictos que en un territorio tan amplio y complejo como el europeo, tienden a multiplicarse. Es aquí donde entran en función los tribunales de justicia que desde hace largo tiempo vienen asumiendo un papel protagonista en el proceso de construcción europea. En particular a partir de la creciente centralidad que desempeña el Tribunal de Justicia de la UE de Luxemburgo tras el desarrollo del mecanismo de las "cuestiones prejudiciales" que permiten resolver las dudas interpretativas que se suscitan en los tribunales de los diferentes Estados.

Un repaso a algunas de las grandes decisiones que han afectado recientemente a nuestro país nos demuestra la trascendencia de su actividad. Los abusos bancarios que estaban en la base de la gran burbuja inmobiliaria que desencadenó la gran crisis, no han encontrado una respuesta definitiva favorable a los propios ciudadanos hasta la resolución del Tribunal de Justicia de la UE (en Luxemburgo) de 21 de diciembre de 2016: con anterioridad, ni las reformas iniciales de la legislación hipotecaria española ni la labor de la propia judicatura nacional habían conseguido resultados tan contundentes e inmediatos. En cuanto al reciente conflicto de la estiba, tiene su base en la decisión de la justicia europea de 11 de diciembre de 2014 contraria a las restricciones a la libre concurrencia que vienen operando desde hace largo tiempo en los puertos españoles. La decisión de que los trabajadores interinos sean indemnizados del mismo modo que los permanentes (Sentencia de 14 septiembre de 2016), ha supuesto toda una revolución en nuestro derecho interno cuyas consecuencias están todavía por desarrollar.

En otros casos se trata de decisiones que afectan al propio núcleo de la Unión: como la defensa de la competencia del presidente del Banco central europeo (BCE), Mario Draghi, para desarrollar su eficaz defensa del euro, frente a las pretensiones de Tribunal federal alemán que intentaban discutir sus atribuciones al respecto (16-VI-2015). O incluso la más reciente decisión de no considerar discriminatoria la prohibición del uso del velo islámico en lugares de trabajo (14-III-2017); una cuestión sobre la que hasta ahora no había una decisión clara y uniforme. 
En resumen, como sucede en los modernos Estados de derecho, la labor de la judicatura se convierte en el instrumento fundamental para defender los derechos humanos y resolver los grandes dilemas interpretativos que plantean sistemas jurídicos complejos: $y$, como viene sucediendo desde hace tiempo, los tribunales europeos se han convertido en la punta de lanza del proyecto europeo.

\section{Balance y conclusión}

Cuando nos formulamos dudas o interrogantes sobre el futuro de la Unión Europea debemos partir de la constatación de que, hasta ahora, la Unión constituye una realidad institucional plenamente consolidada desde hace tiempo: $y$ como sucede con todo sistema institucional consolidado, hay una evidente inercia histórica que empuja a su mantenimiento y auto-reproducción a lo largo del tiempo. Los fines, los valores y los horizontes que se formularon en su origen, y se han reactualizado en diversas renovaciones, seguramente siguen siendo válidos para el conjunto de la sociedad europea.

Por supuesto, si las reacciones estatalistas anti-europeas se siguen reproduciendo en el futuro siguiendo la estela del "brexit", la suerte estará echada. Pero es más que dudoso que en el conjunto de la sociedad europea exista una clara mayoría contraria a la Unión: por eso, seguramente es el momento de constatar sus indudables aciertos, sus avances históricos y sus logros positivos, que han permitido colocar a Europa en una posición estratégica en el nuevo contexto mundial. Con independencia de que las instituciones europeas no hayan conseguido convertirse en una panacea mágica capaz de dar respuestas inmediatas a las grandes crisis desencadenadas en los últimos tiempos.

La reciente cumbre de Roma conmemorando el 60 aniversario de la firma de los pactos fundacionales parece haber optado provisionalmente por un modelo de geometría variable que permita flexibilizar sus pautas de evolución: el futuro dirá si se trata de una estrategia realista y congruente que permita una evolución constructiva del sistema actual.

En el editorial anteriormente citado concluíamos con algunas consideraciones que nos parece oportuno volver a recordar como retos pendientes de la construcción europea: la protección social y la justicia participativa para todos; una economía ecológicamente sostenible y la defensa de esta políitica por la UE en los otros foros internacionales; una atención especial a las mutualidades, cooperativas, otras 
formas de economía social y la inversión ética; una particular atención a la supervisión del sector financiero; la promoción de un consumo responsable; un trabajo conjunto de los países de origen y destino para gestionar de forma regulada los flujos migratorios; un proceso gradual de desarrollo de una auténtica autoridad política mundial, etc.

"Todo esto", decíamos, tiene un origen en los principios que inspiraron el Tratado de Roma, cuyo sexagésimo aniversario conmemoramos. A pesar de lo difícil que era y es llevar adelante estas propuestas por lo que no era tampoco fácil avanzar en el desarrollo del sistema de economía social de mercado, no cejábamos en considerar que ésta era y es el único futuro posible para Europa, si queremos que la UE tenga futuro.

Los rasgos decisivos de la estrategia realista europea con futuro que proponemos podrían resumirse en los siguientes: hacer frente a la competencia global, proporcionando una protección social efectiva compatible con una economía sostenible ambientalmente; desarrollar una comunidad europea de solidaridad; "europeizar" a las generaciones más jóvenes de nuevo en los grandes fundamentos del proyecto europeo, pues hay una generación para la que las razones fundadoras son desconocidas o ya no valen; abordar los desafíos de la interdependencia de las economías nacionales en el ámbito internacional como la comunidad de Estados prósperos e industrializados que es la UE y de esta forma contribuir al desarrollo de una auténtica autoridad política mundial, guiada y regulada por los principios de subsidiariedad y solidaridad y ser expresión de un acuerdo libre y compartido y no el resultado de la coerción o de la violencia, el resultado de un proceso gradual de maduración de la libertad y de la responsabilidad de los pueblos. ${ }^{8}$

Por todo ello, en la conclusión del editorial antes citado", afirmábamos que el patrimonio que está en la raíz del sistema de economía social de mercado debería ser asumido por los cristianos que viven en Europa como algo originario y propio, pues los que refieren su vida y sus convicciones y compromiso desde la caridad, la esperanza y la fe cristianas tienen solidariamente una responsabilidad a la hora de buscar fórmulas concretas para contribuir a la estrategia realista de la construcción de Europa. Fueron cristianos convencidos muchos de los más significativos líderes

\footnotetext{
${ }^{8}$ Cfr. CONSEJO PONTIFICIO JUSTICIA Y PAZ (2011) "Para una reforma del sistema financiero y monetario internacional en la perspectiva de una autoridad pública de ámbito mundial": Revista de Fomento Social 66, 753-771.

${ }^{9}$ Cfr. Consejo de RedACCIÓN (2012) "Para una Europa solidaria...", art. cit. en nota 4.
} 
europeos que estuvieron en el origen del proceso de integración europea. En esta hora de Europa, debe haber ciudadanos comprometidos con el pensamiento y la acción y, en cuanto sean cristianos, mostrarse capaces de

expresar por encima de sus particularidades propias, las exigencias concretas de la fe cristiana para una transformación justa y, por consiguiente, necesaria de la sociedad.

Editoriales publicados sobre Europa en RFS desde $1991^{10}$ :

- Después de Maastricht: más Europa, RFS 47 (1992) n 186.

- Tormenta monetaria sobre Europa, RFS 47 (1992) nº 188.

- La moneda única: imerece la pena? Y después, zqqué?, RFS 52 (1997) n² 205.

- La Política Agraria Común en un mundo globalizado, RFS 56 (2001) nº 223.

- Más allá de la Constitución europea: Europa entre raíces cristianas y laicidad, RFS 59 (2004) n 236.

- La financiación de la Unión Europea: los límites de una ambición, RFS 61 (2006) $n^{\circ} 242$.

- La Constitución europea renace de sus cenizas: el Tratado de Lisboa, RFS 63 (2008) $n^{\circ} 249$.

- "La ilusión de la identidad": el actual debate europeo, RFS 65 (2010) n 257.

- La crisis del euro, RFS 65 (2010) n² 260.

- El futuro de la Política Agraria Común europea: nuevas perspectivas, RFS 66 (2011) n 262

- Un sistema de Economía social de mercado para una Europa responsable, solidaria y productiva, RFS 67 (2012) n 265.

- La política de la Unión Monetaria Europea (UME) y sus consecuencias para España, RFS 67 (2012) n n 268.

- El "ideal realista" europeo. Lo que está en juego en las elecciones al Parlamento europeo, RFS 68 (2013) n 272.

- Una comunidad de memoria y progreso. Francisco en el Parlamento europeo, RFS $69(2014) n^{\circ} 276$.

${ }^{10}$ Cinco editoriales han versado sobre finanzas, cinco sobre la institucionalidad y el sistema constitucional de la UE, tres sobre la identidad cultural y dos sobre la política agraria. 